Tetrahedron Letters No.33, pp. 4017-4023, 1966. Pergamon Press Ltd. Printed in Great Britain.

\title{
ZUR CHEMIE DES ECDYSTERONS
}

H. Hoffmeister und H.F. Grutzmacher

I. Med. Universitätskinik Eppendorf und Chemisches Staatsinstitut, Hamburg

(Received 26 April 1966; in revised form $15 \mathrm{June} 1966$ )

Zwei Häutungs- und Metamorphosehormone der Insekten wurden bisher isoliert: 1954 gelang BUTMNAND darstellung des Ecdysons (1). Die Struktur dieses Häutungshormons konnte von uns aufgeklärt werden $(2,3)$. Es ist ein $2 \beta, 3 \beta, 14 \alpha, 22 \beta_{F}, 25-P e n t a h y d r o x y-5 B$-cholesten-(7)-on-(6). Als ein weiteres Häutungshormon wurde kürzlich Ecdysteron aus Seidenspinnerpuppen rein dargestellt (4). Wir erhielten 9 mg kristallines formon aus 1 to Bombyx mori-Puppen (5).

\section{Struktur und spektroskopische Daten}

Das :lolekulargewicht von Ecdysteron beträgt 480 und nicht wie ursprünglich angegeben ${ }^{*}$ ) 464 . Ein líassenspektrum der hochgereiniften Substanz (6) enthält eine Holekủspitze bei 480 . Tcdysteron hat danach die Summenformel $\mathrm{C}_{27} \mathrm{H}_{44} \mathrm{O}_{7}$. Es unterscreidet sich vom Ecdyson durch eine weitere Hydroxylgruppe, die am C-Atom 20 sitzt. Die Beweise dafür haben wir den spektroskopischen und chemischen Befunden entnommen, die unten wieder Ecdysondaten läßt nur die viederËgebene Deutung zu. tber

*) Das fruher in einein Atlas CH-4-Massenspektrometer erhaltene Spektrum zeigte wegen der thermischen Labilit:t von Ecdysteron keine Molekullionspitze bei 430 , sondern eine Gruppe von Spitzen um 462. 
stereochemische Einzelheiten des Ecdysterons kann nichts ausgesagt werden, jedoch macht die Ähnlichkeit des chemischen Verhaltens und der Spektren die gleichen Ringverknüpfungen sowie die gleiche sterische Anordnung der OH-Gruppen an C-2, $\mathrm{C}-3$ und $\mathrm{C}-14$ in beiden Hormonen wahrscheinlich:
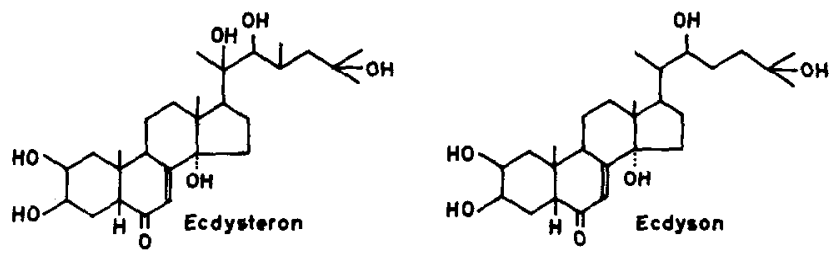

HAMPSHIRE und HOKN gelang jetzt die Isolierung eines Häutungshormons aus Crustaceen (Jasus lalandei), das sie Crustecdyson nannten (7). Das Hormon wurde bisher nicht kristallisiert erhalten, es zeigt biologisch eine weitaus geringere Aktivität als Ecdysteron. Trotzdem lassen die angegebenen Daten vermuten, daß die Substanz mit Ecdysteron identisch ist. Offenbar ist das Hormon noch stärker verunreinigt, worauf auch die geringe Hồhe der UV-Extinktion bei $240 \mathrm{mp}$ schließen läßt ( 8000 gegenüber 13600 beim Ecdysteron).

Das UV-Spektrum von Ecdysteron zeigt in Äthanol ein haximum bei $240 \mathrm{~m} \mu, \varepsilon=13600$. Das IR-ijpektrum des Hormons ist dem des Ecdysons sehr ähnlich. Die Sanden des $\alpha, B$-ungesättigten Ketons haben mit $1645 \mathrm{~cm}^{-1}(C=0)$ und $1612 \mathrm{~cm}^{-1}(C=C)$ die für $\Delta^{7}-6-$ Ketone charakteristische Lage (8). Bei $1383 \mathrm{~cm}^{-1}, 917 \mathrm{~cm}^{-1}$ und 789-843 $\mathrm{cm}^{-1}$ erscheinen Banden, die im Ecdysonspektrum nicht vorhanden aind. 
$\mathrm{Abb} .1$

IR-Spektrum von Ecdysteron in KBr. Der KBr-Pressling wurde mit IR-Licht getempert.

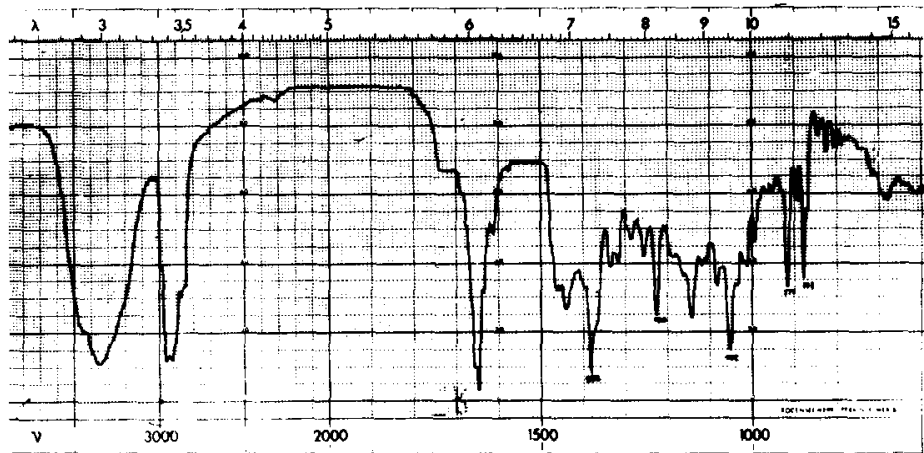

Tab. 1

$\delta$-Werte der Signale im Frotonenresonanzspektrum des Ecdysterons.

\begin{tabular}{|c|c|c|}
\hline$\delta$-Werte (ppm) & Anzahl der Protonen & Funktion \\
\hline 1,06 & 3 & C-19-Methylgruppe \\
1,19 & 3 & C-18-Methyl Gruppe \\
1,34 & 6 & C-26/27-Methylgruppen \\
1,55 & 3 & C-21-Methy gruppe \\
6,16 & 1 & H-Atom an C-7 \\
\hline
\end{tabular}

Im Frotonenresonanzspektrum des Ecdysterons liegen die Signale der Isopropanol-Endgruppe der Seitenkette sowie der C-19-Methylgruppe an fiast der gleichen Stelle wie im Ecdysonspektruin. Das Duilett der C-21-Methylgruppe des Icdysons tehlt im Ecdysteronspektrun; dafur taucht ein scharfes Signal von 3 Frotonen bei 1,55 ppm aut. Die starke Verschiebung des c-21-Methylsignals und die Imwandlune des Dubletts in ein singulett ist nur zu erklären durch den Eintritt einer OHiruppe an C-20. Als odellsuustanzen murden von uns 27-Nor-5 $\alpha-$ cholestan-diol-(3B,20)-on-(25)-3-acetat-ätinylenacetal und $5 \alpha-$ Cnolestan-diol- $(3[3,25)-3-$ monoacetat hergestellt (8). Die 
in Pyridin aufgenomenen Protonenresonanzspektren dieser Substanzen zeigen Verschiebungen, die mit den bei Ecdyson/ Ecdysteron gefundenen sehr gut ubereinstimmen:

Tab. 2

Verschiebung der C-21- und C-18-Methylgruppensignale im NMRSpektrum durch eine OH-Gruppe in Stellung 20 . $\delta$-Werte (ppm).

\begin{tabular}{|c|c|c|c|}
\hline Verbindung & $C-21$ & $C-18$ & Differenz \\
\hline Ecdyson & Dublett $1,34 / 1,22$ & 0,74 & \multirow{2}{*}{0,45} \\
\hline Ecdysteron & Singulett 1,55 & 1,19 & \\
\hline $\begin{array}{l}27-\text { Nor }-5 \alpha-\text { cholestan- } \\
\text { diol- }(3 \beta, 20)-\text { on- }(25)- \\
\text { 3-acetat-athylen- } \\
\text { acetal }\end{array}$ & Dublett $0,95 / 1,04$ & 0,67 & \multirow{2}{*}{0,40} \\
\hline $\begin{array}{l}5 \alpha-\text { Cholestan-diol- } \\
(3 B, 25)-3-\text { mono- } \\
\text { acetat }\end{array}$ & Singulett 1,46 & 1,07 & \\
\hline
\end{tabular}

Einen weiteren Deweis für die stellung der 6.Hydroxylgruppe in Ecdysteron erhält man aus der Iage der C-18-Methylgruppe. Während das Signal in Ecdysonspektrum mit 0,74 ppm einen von Substituenten unbeeinflußten Wert zeigt, liegt das entsprechende Signal des Ecajsterons weit verschoben bei 1,19 ppm. Die Verschiebung un den annähernd gleichen Betrag findet man auch bei den Vergleichssubstanzen in Tabelle 2 .

Das Massenspektrum von Ecdysteron (Abb.2) enthält die Peaks vom Molekulion und einer vierfachen Wasserabspaltun $(480,462,444,426,408)$. Die Eliminierune von vier Wassermolekilen aus den Ecdysteron in Gegensatz zur suspaltung von nur drei Molekulen beim Ecdyson (2) macht ebenfalls deutlich, daß Eodysteron eine Oi-Gruppe menr enthät. 
$A b b \cdot 2$

Massenspektrum des Ecdysterons (Atlas CH 4-Gerät, Verdampfungstemperatur $150 \mathrm{C}$, Elektronenenergie $70 \mathrm{eV}$ ).

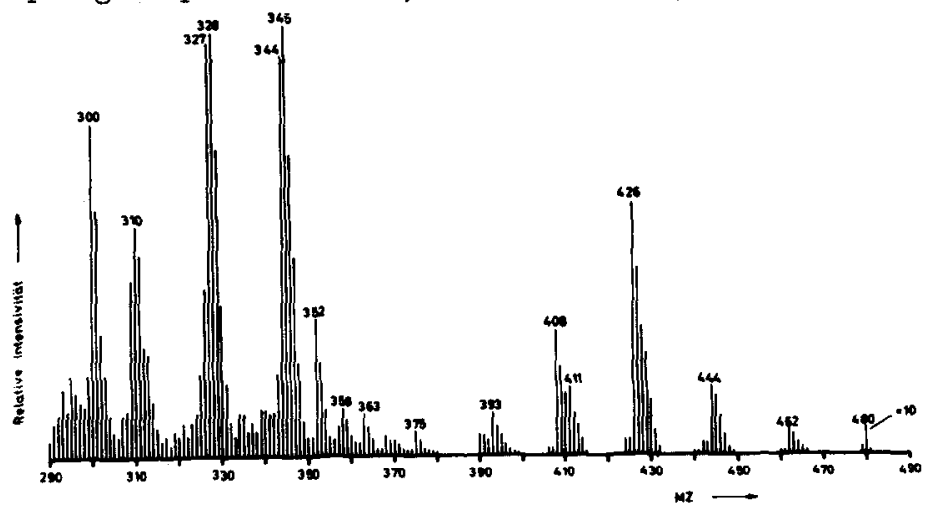

Der Hauptpeak liegt wie im Massenspektrum des Ecdysons bei MZ 99: Dieses Ion entsteht wie dort durch Spaltung der Seitenkette zwischen $\mathrm{C}-20$ und $\mathrm{C}-22$ (2). Das beweigt, daB Ecdysteron und Ecdyson ab C-22 die gleiche Seitenkette besitzen.<smiles>CC(C)CC(O)C(C)(C)C1CCC(C)C1(C)C</smiles>

Aus den Bruchstücken MZ 426 und MZ 408 wird jeweils eine anguIare Methylgruppe abgespalten (MZ 411, MZ 393), wie es typisch ist für Steroide (9). In oberen líassenbereich sind erhebliche Unterschiede zum Ecdysonspektrum vorhanden. Der fiur Ecdyson charakterlstische Peak 330 fehlt, statt dessen tritt ein groBer Peak liz 345 auf. Das Ion liZ 330 entsteht aus dem Ecdyson über ein Ion $\mathrm{NZ} 348$ durch spaltung der Bindung C-20/C-22 unter Wanderung eines H-Atoms und anschlieBender $\mathrm{H}_{2} \mathrm{O}$-Abspaltung (2). Da die zusätzliche Hydroxylgruppe des Ecdysterons in diesem Teil des Ifolekuls enthalten sein muß, wären bei einem analogen Zerfall Peaks mit den MZ 364 und MZ 346 zu er- 
warten. Das Massenspektrum zeigt aber in diesem Bereich Gruppen von Peaks vergleichbarer Intensität, aus denen die Wassen. zahlen 363,345 und 328 hervorragen. Daraus folgt, daB im Gegensatz zum Ecdyson die Spaltung der C-20/C-22-Bindung und Bildung der Ionen $\mathrm{NZ} 363$ bzw. 345 ohne Wanderung eines H-Atomk ablauft. Dies Verhalten wird verständich, wenn die zusätzlicile $\mathrm{OH}-G$ ruppe an C-20 lokalisiert ist; eine positive Ladung an C-20 wird durch Nesomerie mit der Hydroxylgruppe stabilisiert.

Die angenominene Stelling der IIydroxylgruppe wird ebenfalls gestützt durch den Peak MZ 300, der mit vergleichbarer Intensität in den Spektren beider Hormone vorharden ist. Für dieses Ion kann angenommen werden, daß es durch verlust der voll- . städiEen Seitenkette an $\mathrm{C}-17$, eines $\mathrm{H}_{2} \mathrm{O}-\mathrm{Holekiuls}$ und eines $\mathrm{H}-\mathrm{A}$ toms entstanden ist.

\section{Säureabpau}

Ecdysteron wird wie Ecdyson durch Säuren schnell umgewandelt in bioloriscin inaktive Substanzen. In Fall des Rcdysons konnten wir die mit Salzsäure entstandenen Unwandlungsprodukte aufklären und durch UV-spektren charakterisieren (2). Gibt man zu einer aịkoholischen Lösung von Ecdysteron $1 ; 5 \mathrm{n} \mathrm{HCl}$, dann fällt die Extinktion bei $240 \mathrm{mp}$ nach $5 \mathrm{~h}$ aut die Hälfte. Daftir bildet sich ein entsprechendes Maximum bei $293 \mathrm{mp}$ aus. Nach weiteren $15 \mathrm{~h}$ Stehen bei zimmertemperatur verliert sich das Maximum bei $293 \mathrm{~m} \mu$ und ein neues Maximum bei $244 \mathrm{mp}$ taucht auf. Diese Umwandlungen des Ecdysterons sind inentisch mit den bei Ecdyson gefundenen. Es haridelt sich um die charakteristische Abspaltung der 18-OH-Gruppe unter Ausbildung eines 
$\triangle 7,14(15)$-Dien-on(6)-Systems und anschlieBende Umlagerung in das stabilere $\Delta^{8,14}$-Dien-on-(6).

Der Deutschen Forschungsgemeinschaft danken wir für die Unterstitzung dieser Arbeit.

\section{Literaturverzeichnis}

1) A.Butenandt u. P.Karlson, Z.Naturforsch. 2b, 389 (1954)

2) P.Karlson, H.Hoffmeister, H.Hummel, P.Hocks u. G.Spiteller, Chem.Ber. 98, 2394 (1965)

3) R.Huber u. W.Hoppe, Chem.Ber. 28, 2403 (1965)

4) H.Hoffmeister, Angew. Chemie 78, 269 (1966)

5) H.Hoffmeister, Z.Naturforsch. 21b, 335 (1966)

6) H.Hoftreister U.H.F.Grützmacher, Z.Naturtorsch. (imDruck)

7) F.Hampshire u. D.H.S.IJorn, Chem.Communic. 2, 37 (1966)

8) H.Hoffneister, C.Zufer, H.H.Keller, H.Wchairer u. P.Karlson, Chem.Ber. 28, 2361 (1965)

9) H.Budzikiewice, C.Derassi, D.H.villiams, Structure Elucidation of Vatural Products By Uss Spectrometry, Vol 2, Holden-Day Inc. 1964 